\title{
The Effect of Dry Needling on Range of Motion of Neck Lateral Flexion in Subjects With Active Trigger Point in Upper Trapezius Muscle
}

\author{
Maryam Ziaeifar ${ }^{1}$; Amir Massoud Arab ${ }^{1,{ }^{*}}$; Noureddin Karimi ${ }^{1}$; Zahra Mosallanejad ${ }^{1}$ \\ ${ }^{1}$ Department of Physical Therapy, University of Social Welfare and Rehabilitation Sciences, Tehran, IR Iran \\ *Corresponding author: Amir Massoud Arab, Department of Physical Therapy, University of Social Welfare and Rehabilitation Sciences, Evin, Koodakyar Ave., P.O. Box: 1985713834, \\ Tehran, IR Iran. Tel/Fax: +98-2122180039, E-mail:arabloo_masoud@hotmail.com
}

Received: September 4, 2014; Revised: October 10, 2014; Accepted: November 15, 2014

\begin{abstract}
Background: Myofascial trigger point is one of the most common causes of musculoskeletal disorders. Myofascial trigger point in upper trapezius has been reported as a symptom in patients with neck and upper thoracic pain.

Objectives: The purpose of this study was to investigate the effect of dry needling compared with ischemic pressure on trigger point in upper trapezius muscle.

Materials and Methods: 28 subjects with myofascial trigger point in upper trapezius participated in this study. The subjects were randomly assigned to two groups: dry needling $(n=13)$ and ischemic pressure $(n=15)$. The neck lateral flexion range of motion was measured before and after treatment in both groups using a standard goniometer. Paired t-test was used to determine any significant difference in range of motion after treatment sessions compared with pre-treatment score in control and experimental group. Analysis of Covariance (ANCOVA) was calculated to determine the significance of differences between the control and experimental groups in posttest scores, with pre-treatment scores used as covariates in the analysis.

Results: Statistical analysis (paired t-test) revealed significant increase in neck lateral flexion range of motion in contra-lateral side after treatment sessions in control and experimental group compared with pre-treatment score $(\mathrm{P}<0.05)$. However, only dry needling was effective in increase of range of motion in Ipsi-lateral side $(\mathrm{P}=0.001)$. In the ANCOVA, controlling for pre-test scores, no significant difference was found between the two groups in the after treatment sessions $(P=0.06)$ and $(P=0.15)$.

Conclusions: The application of DN produces an improvement in ILF and CLF can be prescribed for subjects with MTP in UT muscles.
\end{abstract}

Keywords: Myofascial Pain Syndromes; Superficial Back Muscles; Ischemia; Pressure

\section{Background}

Musculoskeletal pain is a major cause of morbidity in today's societies (1-3). About one-third of the patients with musculoskeletal pain meet the diagnostic criteria for myofascial pain syndrome (1). A myofascial trigger point (MTP) has been explained as a hyperirritability spot located in a taut band of muscle; described as resembling a small pea or as a rope-like nodular or crepitant (crackling, grating) area within the muscle which is painful in palpation or compression and refers pain, tenderness, or an autonomic response to a remote area. Some investigators stated that when pressure is applied to a MTP, a "jump sign" or "jump response" is elicited whereby the patient reacts with facial grimacing or by jumping away from the examiner (4).

Previous studies have indicated that MTPs are the primary source of musculoskeletal pain in $21-85 \%$ of patients $(5,6)$. The prevalence of MTP varies from $21 \%$ of patients seen in a general orthopedic clinic, to $30 \%$ of general medical clinic patients with regional pain, to as high as $85 \%$ to $93 \%$ of patients presenting to specialty pain management centers $(7,8)$. It has detrimental effects on people's social and work-related activities and has a significant impact on the quality of life and causes pain and functional disability in neck and shoulder area $(2,3,9)$.

It is thought that MTP may result from or be irritated by trauma, overuse, mechanical overload, postural faults, or psychological stress (4). Considering the sedentary lifestyle in today's societies, as one spends much time in static postures, phasic muscles will become progressively inhibited and lax while postural muscles are gradually tightened. A muscle imbalance between the dynamic and postural muscles may lead to MTP in lumbo-pelvic or cervical region (3).

Some chemical changes such as increased level of bradykinin, substance $\mathrm{P}$, and calcitonin gene-related peptide (CGRP), and lowered pH has been shown in MTP $(10,11)$. Investigators established that the local oxygen saturation at a MTP site is less than 5\% of normal. Hypoxia leads to the release of local release of several nociceptive chemicals, including bradykinin, CGRP, and substance P (10). Local tenderness and referred pain following MTP ensues as muscle nociceptors are stimulated in response to reduced oxygen levels and increased inflammatory chemicals $(6,10,12,13)$.

Copyright (C) 2014, Ahvaz Jundishapur University of Medical Sciences. This is an open-access article distributed under the terms of the Creative Commons Attribution-NonCommercial 4.0 International License (http://creativecommons.org/licenses/by-nc/4.0/) which permits copy and redistribute the material just in noncommercial usages, provided the original work is properly cited. 
Histological studies have confirmed the presence of extreme sacromere contractions, resulting in localized tissue hypoxia (10). The combination of increased levels of CGRP and lowered pH suggest that the MTP site is too acidic for ACh to work efficiently. It has been hypothesized that the injured muscle fibers shorten (making taut bands) either in response to excessive amounts of calcium ions being released from within the damaged fibers, or in response to the corresponding motor end plate releasing excessive amounts of $\mathrm{ACh}(6,10,13)$.

The upper trapezius (UT) muscle was found by investigators to be the muscle most often affected by MTP (1416). The common symptoms and pain pattern in subjects with MTP in UT muscle is taut and painful muscle, tension headache, neck pain, dizziness or vertigo, limited neck and shoulder ROM (4,17-19).

Considering the attachment of the UT muscle to cervical spine and suboccipital region and with regard to the role of UT in controlling range of motion (ROM) of the cervical lateral flexion, it is thought that MTP in UT muscle can result in limited cervical ROM (20). Local twitch response (LTR); an spontaneous spinal cord reflex contraction of the taut band muscle fibers in response to palpation or needling, is one of the most important characteristics of $\operatorname{MTP}(5,10,21)$.

Physical therapy programs play a significant role in treatment and improvement of symptoms in patients with MTP. The ischemic compression (IC) is currently used for the subjects with MTP attending physical therapy clinics $(1,13,22)$. Dry needling $(D N)$ has been recently used by therapists to treat MTP. The objectives of DN are achieved by inactivating the MTP, normalizing the chemical environment of active MTP, releasing muscle shortening, removing the source of muscle irritation, normalizing peripheral nerve sensitization, promoting self-healing of the injured tissue and decreasing spontaneous muscle activity (10).

Investigators have attributed the therapeutic effects of DN to various mechanisms, such as mechanical, neurophysiologic and chemical effects (10). It is thought that DN mechanically provides a localized stretch to the shortened sarcomeres and contracted cytoskeletal structures within the MTP. This would allow the sarcomere to resume its resting length by reducing the degree of overlap between actin and myosin filaments $(5,10,21)$.

In favor of neurophysiological explanations of the DN effects, it has been suggested that DN stimulates the A-delta nerve fibers (group III), which in turn, may activate the enkephalinergic inhibitory dorsal horn interneurons, resulting in opioid mediated pain suppression (Pain relief) $(10,21)$. For chemical effect of DN, some studies have demonstrated that the increased levels of bradykinin, CGRP, substance P, and other chemicals at MTP are directly corrected by eliciting LTR following DN (10).

It has been suggested that DN may influence the microcirculation. Several investigators have demonstrated that needle insertion in the muscles increased both skin and muscle blood flow in the stimulated region (23). A change in inflammatory mediators has been demonstrated after DN of the UT, which suggested increasing local blood flow to the MTP region $(23,24)$. Although some previous studies have assessed the effect of DN on MTP in UT, review of the literature showed that a few RCT (randomized clinical trials) have been conducted to determine the effectiveness of DN in the treatment of MTP in UT muscle $(25,26)$.

However, with the use of different designs, samples and testing procedures, controversial results have been reported regarding the effect of DN on MTP in UT. Some previous studies assessed the immediate effect of DN, some others investigated the efficacy of DN combined with other modalities and some others investigated the remote effect of DN on UT muscle. Review of the literature showed that a few RCT (randomized clinical trials) have been conducted to determine the effectiveness of DN alone in the treatment of MTP in UT muscle (23, 25-29).

\section{Objectives}

The purpose of this study was to investigate the effect of $\mathrm{DN}$ on active cervical lateral flexion range of motion compared with IC in individuals with MTP in UT muscle.

\section{Materials and Methods}

A total of 28 patients with MTP in UT muscle, who had been referred for outpatient physical therapy evaluation and intervention, participated in this study (IC = $15, \mathrm{DN}=13$ ). The patient population in this study was a sample of convenience made up of subjects who were between the ages of 19 and 48 years. They were consecutive patients who agreed to participate and fulfilled the inclusion criteria.

The inclusion criteria for having active MTP in UT muscle was as follow $(30,31)$ :

1- Presence of palpable taut band in muscle.

2- Presence of a hypersensitive tender spot in the taut band.

3- Reproduction of the typical referred pain pattern of the MTP in response to compression. For third criteria (detecting active MTP), MTP pressure tolerance was assessed using a mechanical pressure algometer. The investigator applied continuous pressure with the algometer at approximately about a pressure of $2.5 \mathrm{~kg} / \mathrm{cm}^{2}$.

4- Spontaneous presence of the typical referred pain pattern and/or patient recognition of the referred pain as familiar.

5- Pain of at least $30 \mathrm{~mm}$ on a visual analogue scale (VAS).

The selected MTP of the UT muscle was located in the middle of the more nearly horizontal fibers of the UT (29).

Patients were also excluded if they had a history of fibromyalgia syndrome, whiplash injury, cervical spine surgery and fracture, cervical radiculopathy, having MTP therapy within the past month before the study and any systematic disease such as rheumatism and tuberculosis or cervical myelopathy, multiple sclerosis (12, 32-35). The 
patients, who underwent DN, also had no contraindication for needling such as local infection, pregnancy with threatened abortion, taking anticoagulants (e.g. warfarin) and long-term steroid using. After the initial screening, 33 patients fulfilled all inclusion criteria. Before participating in the study, all subjects signed an informed consent form approved by the human subjects committee at the University of Social Welfare and Rehabilitation Sciences.

\subsection{Assignment}

Patients were randomly assigned to a control (IC) group ( $n=15$, mean age $=26.5 \pm 8.57$ years $)$ and an experimental (DN) group $(n=13$, mean age $=29.8 \pm 10.71$ years $)$. Physical characteristics of the patients in each group are shown in Table 1.

\subsection{Procedure}

The procedure for both groups was as follow:

The treatment protocol for control group consisted of the IC technique on MTP in UT muscle. The patients in the experimental group received DN for UT muscle. For the ischemic compression technique, the patient was in supine or prone with the cervical spine in a neutral position. The therapist applied gradually increasing pressure to the MTP until the sensation of pressure and pain. At that moment, the pressure was maintained until the discomfort and/or pain relieved by around 50\%, perceived by the own patient. At that time, the pressure was increased until discomfort appeared again. This process was repeated for 90 second. $(31,32)$.

The treatment protocol for experimental group consisted of the DN technique on MTP in UT muscle. DN for MTP was performed as described by others. For this purpose; the subject was asked to lie in prone position. The taut band; localized between the thumb and index finger, was needled forward and backward repeatedly until there were no more LTR (25). The outcome measures were measured before treatment and after treatment sessions (one week). The treatment frequency was three times per week.

\subsection{Outcome Measures}

The active ipsilateral flexion (ILF) ROM and contralateral lateral flexion (CLF) ROM of the cervical spine were collected before treatment and after treatment sessions in both groups.

\subsection{Assessment of Lateral Flex}

The ROM of the ILF and CLF of the cervical spine were measured using a universal goniometer. For this purpose, the subject was in sitting position with thoracic and lumbar spine well supported by the back of the chair and the cervical spine is in 0 degree of flexion/extension and rotation. Wooden tongue depressor was held between teeth for reference. The goniometer fulcrums were alongside the tongue depressor and the cervical lateral flexion range of motion was measured (36).

\subsection{Statistical Analysis}

Statistical analysis was performed using SPSS version 17.0. Paired t-test was used to determine any significant change in ILF and CLF after treatment sessions compared with pre-treatment score in control and treatment group. Analysis of covariance (ANCOVA) was calculated to determine the significance of differences between the control and experimental groups in post-test measurements.

\section{Results}

Demographic data (Mean \pm SD) for the subjects in both groups is presented in Table1. Pre-and post-measurement scores for active ILF and CLF of the cervical ROM in the control and intervention group and the results of paired t- test are provided in Table 2. The mean ILF ROM score increased from 39.33 and 41.26 pre-test to 47.5 and 44.46 post-test respectively for DN and IC group. The mean CLF ROM scores increased from 38.5 and 39.33 before treatment to 46.66 and 43.4 after treatment respectively in DN and IC group.

The result of paired t-test revealed a significant change in CLF after treatment in DN and IC group compared with before treatment $(\mathrm{P}<0.05)$ but a significant change in ILF after treatment only in DN group compared with before treatment $(\mathrm{P}<0.05)$. The result of ANCOVA revealed no significant difference was found between the two groups on the post-measurement score of $\operatorname{ILF}(\mathrm{P}=0.06)$, and CLF $(\mathrm{P}=0.15)$.

\begin{tabular}{ccc}
\hline \multicolumn{3}{l}{ Table 1. Demographic Data of the Subjects ${ }^{\text {a }}$} \\
\hline Variables & $\begin{array}{c}\text { Control Group } \\
(\mathbf{n}=\mathbf{1 7})\end{array}$ & $\begin{array}{c}\text { Experimental Group } \\
(\mathbf{n}=\mathbf{1 6})\end{array}$ \\
\hline Age, $\mathbf{y}$ & $26.5 \pm 8.57$ & $30.06 \pm 9.87$ \\
Weight, $\mathbf{~ k g}$ & $56 \pm 5.92$ & $60.37 \pm 6.96$ \\
Height, $\mathbf{c m}$ & $163.7 \pm 4.49$ & $165.3 \pm 7.56$ \\
\hline
\end{tabular}

a Data are presented as mean \pm SD.

Table 2. Pre- and Post-Measurement Scores for ILF and CLF in the Subjects $\mathrm{a}$, b

\begin{tabular}{cccc}
\hline Variables & $\begin{array}{c}\text { Before } \\
\text { Treatment }\end{array}$ & $\begin{array}{c}\text { After } \\
\text { Treatment }\end{array}$ & P Value \\
\hline ILF & & & \\
DN & $39.33 \pm 6.63$ & $47.5 \pm 5.83$ & 0.001 \\
IC & $41.26 \pm 9.66$ & $44.46 \pm 7.55$ & 0.08 \\
\hline CLF & & & \\
\hline DN & $38.5 \pm 8.35$ & $46.66 \pm 6.51$ & 0.005 \\
\hline IC & $39.33 \pm 8.85$ & $43.4 \pm 8.6$ & 0.04 \\
\hline
\end{tabular}

\footnotetext{
${ }^{a}$ Abbreviations: CLF, contera lateral flex; DN, Dry needling; IC, ischemic compression; ILF, ipsi lateral flex.

$\mathrm{b}$ Data are presented as mean $\pm \mathrm{SD}$.
} 
Table 3. The Results of ANCOVA in Control and Experimental Groups ${ }^{\mathrm{a}}$

\begin{tabular}{lccccc}
\hline Variable & $\begin{array}{c}\text { Sum } \\
\text { square }\end{array}$ & DF & $\begin{array}{c}\text { Mean } \\
\text { square }\end{array}$ & F & P Value \\
\hline ILF & 106.5 & 1 & 106.5 & 3.64 & 0.06 \\
CLF & 90.94 & 1 & 90.94 & 2.17 & 0.15 \\
\hline
\end{tabular}

a Abbreviations: CLF, contera lateral flex; ILF, ipsi lateral flex.

\section{Discussion}

The result of this study is in agreement with the study conducted by Dominic et al who showed the increase in cervical ROM after needling (27). Limitation in ROM is one of the most important strategies in the human body to decrease the muscular injury. This ROM limitation is accompanied by muscle stiffness especially in chronic musculoskeletal syndromes (20). In today's sedentary lifestyle, peoples are in static position for long time duration. Postural muscles like UT tend to be tightened. Muscle imbalance between postural and phasic muscles results in myofascial pain syndromes. Tightness in postural muscles can induce limited ROM. Thus, myofascial pain syndromes may present in the forms of pain, decreased joint motion, and muscle tightness or spasm (4).

Pain signal transferred to the spinal cord induces a nociceptive reflex. Continuous nociceptive reflex results in sustained muscle spasm (pain/spasm cycle). Continuous spinal stimulation and sustained muscle contraction results in muscle shortening and trigger point formation. Tissue hyper-sensitivity and reflexive shortening in MTP can increase mechanical pressure on the nerve endings. Released substance P from the nerve endings increases tissue sensitivity and excitability. Increased tissue excitability causes sustained muscle contraction in response to normal mechanical stimulants. Continuous muscle contraction leads to muscle spasm and pain. Prolonged local spasm results in muscle fiber shortening and tightness. Shortened muscle fibers will limit the joint ROM (10). DN may mechanically disrupt the integrity of the hyper tone muscle fibers - trigger points. Inserted needle can provide a localized stretch to the muscle fibers and can induce LTR which can cause muscle relaxation through reflex inhibition. Muscle relaxation can improve the $\operatorname{ROM}(10,21)$.

The result of this study showed a significant change in CLF after treatment sessions compared with pre-treatment score in control and experimental group and a significant change in ILF after treatment sessions compared with pre-treatment score only in experimental group. Our data showed no significant difference in increasing ILF and increasing CLF score between two groups after treatment. The fact that the experimental group showed a significantly greater improvement in ILF and CLF after treatment, may suggest that the application of DN could be considered as a therapy of choice for subjects with MTP in UT muscle.
This finding is in accordance with other studies showing effects of DN in patients with MTP in UT muscle. However, with the use of different designs and testing procedures, controversial results have been reported regarding the effect of DN on MTP. Most of the previous studies assessed the immediate effect of DN in MTP. Some others investigated the efficacy of DN combined with other modalities and some others investigated the remote effect of DN on UT muscle (25-29). Review of the literature showed that a few RCT (randomized clinical trials) have been conducted to assess the efficacy of DN in the treatment of MTP in UT muscle (25-29). To our knowledge, this is the first study that directly investigated the effect of DN on MTP after one week. Investigators have attributed the therapeutic effects of DN to mechanical, neurophysiologic and chemical effects (10). Some chemical and mechanical changes such as increased level of bradykinin, substance P, and CGRP, and lowered $\mathrm{pH}$, hypoxia, local tenderness and decrease in PPT has been shown in MTP $(10,11)$. Local tenderness and referred pain following MTP ensues as muscle nociceptors are stimulated in response to reduced oxygen levels and increased inflammatory chemicals $(6,10,12,13)$.

The results' following the application of DN is reflected in the work of some other researchers who studied the mechanical, neurophysiologic and chemical effects of the tissue in response to the $\mathrm{DN}(10)$. $\mathrm{DN}$ mechanically provides a localized stretch to the shortened sarcomeres which allow the sarcomere to resume its resting length by reducing the degree of overlap between actin and myosin filaments (5, 10,21 ). It has been also suggested that DN stimulates the Adelta nerve fibers which in turn may activate the enkephalinergic inhibitory dorsal horn interneurons $(10,21)$. Some studies have demonstrated an increased levels of bradykinin, CGRP, substance P, and other chemicals at MTP combined with eliciting LTR following DN (10).

Our data, however, showed no significant difference in increasing ILF and CLF between two groups after treatment. The reason we found no statistical differences between groups can be explained by the relatively small between group differences observed. As shown, the maximum between group change scores for ILF scores was 4.97 and the maximum between group change scores for CLF scores was 4.09 which was not statistically different. However, this difference could be considered clinically important and it is possible that if the sample size had been larger. The application of DN produces an improvement in ILF and CLF can be prescribed for subjects with MTP in UT muscles.

\section{Authors' Contributions}

All authors has been contributed in research, drafting, writing and editing the manuscript equally.

\section{Funding/Support}

Partially supported by the University of Social Welfare and Rehabilitation Sciences. 


\section{References}

1. Rickards LD. The effectiveness of non-invasive treatments for active myofascial trigger point pain: A systematic review of the literature. Int J Osteopath Med. 2006;9(4):120-36.

2. Tough EA, White AR, Richards S, Campbell J. Variability of criteria used to diagnose myofascial trigger point pain syndrome-evidence from a review of the literature. Clin J Pain. 2007; 23(3):278-86.

3. Yap EC. Myofascial pain--an overview. Ann Acad Med Singapore. 2007;36(1):43-8.

4. Hanten WP, Olson SL, Butts NL, Nowicki AL. Effectiveness of a home program of ischemic pressure followed by sustained stretch for treatment of myofascial trigger points. Phys Ther. 2000;80(10):997-1003.

5. Rickards LD. Therapeutic needling in osteopathic practice: An evidence-informed perspective. Int J Osteopath Med. 2009;12(1):2-13.

6. Tough EA, White AR, Cummings TM, Richards SH, Campbell JL. Acupuncture and dry needling in the management of myofascial trigger point pain: a systematic review and meta-analysis of randomised controlled trials. Eur J Pain. 2009;13(1):3-10.

7. Borg-Stein J, Simons DG. Focused review: myofascial pain. Arch Phys Med Rehabil. 2002;83(3 Suppl 1):S40-7.

8. Shah JP, Danoff JV, Desai MJ, Parikh S, Nakamura LY, Phillips TM, et al. Biochemicals associated with pain and inflammation are elevated in sites near to and remote from active myofascial trigger points. Arch Phys Med Rehabil. 2008;89(1):16-23.

9. Cummings M, Baldry P. Regional myofascial pain: diagnosis and management. Best Pract Res Clin Rheumatol. 2007;21(2):367-87.

10. Dommerholt J. Dry needling in orthopedic physical therapy practice. Orthop Phys Ther Pract. 2004;16(3):15-20.

11. Shah JP. Integrating Dry Needling with New Concepts of Myofascial Pain, Muscle Physiology, and Sensitization. In: Audette JF, Bailey A editors. Integrative Pain Medicine.: Humana Press; 2008. pp.107-21.

12. Hsieh YL, Kao MJ, Kuan TS, Chen SM, Chen JT, Hong CZ. Dry needling to a key myofascial trigger point may reduce the irritability of satellite MTrPs. Am J Phys Med Rehabil. 2007;86(5):397-403.

13. Chaitow L, Fritz S, King RK, Chambers G. A massage therapist's guide to understanding, locating and treating myofascial trigger points.: Churchill Livingstone/Elsevier; 2006.

14. Sarrafzadeh J, Ahmadi A, Yassin M. The effects of pressure release, phonophoresis of hydrocortisone, and ultrasound on upper trapezius latent myofascial trigger point. Arch Phys Med Rehabil. 2012;93(1):72-7.

15. Sciotti VM, Mittak VL, DiMarco L, Ford LM, Plezbert J, Santipadri $\mathrm{E}$, et al. Clinical precision of myofascial trigger point location in the trapezius muscle. Pain. 2001;93(3):259-66.

16. Gemmell H, Allen A. Relative immediate effect of ischaemic compression and activator trigger point therapy on active upper trapezius trigger points: A randomised trial. Clin Chiropr. 2008;11(4):175-81.

17. Lucas N, Macaskill P, Irwig L, Moran R, Bogduk N. Reliability of physical examination for diagnosis of myofascial trigger points: a systematic review of the literature. Clin J Pain. 2009;25(1):80-9.

18. Rudin NJ. Evaluation of treatments for myofascial pain syndrome and fibromyalgia. Curr Pain Headache Rep. 2003;7(6):433-42.

19. Fernandez-de-las-Penas C, Alonso-Blanco C, Miangolarra JC. Myofascial trigger points in subjects presenting with mechanical neck pain: a blinded, controlled study. Man Ther. 2007;12(1):29-33.

20. Lederman E. Neuromuscular rehabilitation in manual and physical therapies: principles to practice.: Churchill Livingstone; 2010.

21. Dommerholt J, del Moral OM, Grobli C. Trigger point dry needling. Myofascial Trigger Points: pathophysiology and evidence-informed diagnosis and management.: Jones \& Bartlett Publishers; 2009.

22. de las Penas CF, Sohrbeck Campo M, Fernandez Carnero J, Miangolarra Page JC. Manual therapies in myofascial trigger point treatment: a systematic review. J Body Mov Ther. 2005;9(1):27-34.

23. Cagnie B, Barbe T, De Ridder E, Van Oosterwijck J, Cools A, Danneels L. The influence of dry needling of the trapezius muscle on muscle blood flow and oxygenation. J Manipulative Physiol Ther. 2012;35(9):685-91.

24. Shah JP, Gilliams EA. Uncovering the biochemical milieu of myofascial trigger points using in vivo microdialysis: an application of muscle pain concepts to myofascial pain syndrome. J Bodyw Mov Ther. 2008;12(4):371-84.

25. Ga H, Choi JH, Park CH, Yoon HJ. Dry needling of trigger points with and without paraspinal needling in myofascial pain syndromes in elderly patients. J Altern Complement Med. 2007;13(6):617-24.

26. Ilbuldu E, Cakmak A, Disci R, Aydin R. Comparison of laser, dry needling, and placebo laser treatments in myofascial pain syndrome. Photomed Laser Surg. 2004;22(4):306-11.

27. Irnich D, Behrens N, Gleditsch JM, Stor W, Schreiber MA, Schops $\mathrm{P}$, et al. Immediate effects of dry needling and acupuncture at distant points in chronic neck pain: results of a randomized, double-blind, sham-controlled crossover trial. Pain. 2002;99(12):83-9.

28. Edwards J, Knowles N. Superficial dry needling and active stretching in the treatment of myofascial pain--a randomised controlled trial. Acupunct Med. 2003;21(3):80-6.

29. Tsai CT, Hsieh LF, Kuan TS, Kao MJ, Chou LW, Hong CZ. Remote effects of dry needling on the irritability of the myofascial trigger point in the upper trapezius muscle. Am J Phys Med Rehabil. 2010;89(2):133-40.

30. Aguilera FJ, Martin DP, Masanet RA, Botella AC, Soler LB, Morell FB. Immediate effect of ultrasound and ischemic compression techniques for the treatment of trapezius latent myofascial trigger points in healthy subjects: a randomized controlled study. $J$ Manipulative Physiol Ther. 2009;32(7):515-20.

31. Gemmell H, Miller P, Nordstrom H. Immediate effect of ischaemic compression and trigger point pressure release on neck pain and upper trapezius trigger points: A randomised controlled trial. Clin Chiropr. 2008;11(1):30-6.

32. Fernandez-de-las-Penas C, Alonso-Blanco C, Fernandez-Carnero J, Carlos Miangolarra-Page J. The immediate effect of ischemic compression technique and transverse friction massage on tenderness of active and latent myofascial trigger points: a pilot study.J Bodyw Mov Ther. 2006;10(1):3-9.

33. Blikstad A, Gemmell H. Immediate effect of activator trigger point therapy and myofascial band therapy on non-specific neck pain in patients with upper trapezius trigger points compared to sham ultrasound: A randomised controlled trial. Clin Chiropr. 2008;11(1):23-9.

34. Ruiz-Saez M, Fernandez-de-las-Penas C, Blanco CR, MartinezSegura R, Garcia-Leon R. Changes in pressure pain sensitivity in latent myofascial trigger points in the upper trapezius muscle after a cervical spine manipulation in pain-free subjects. $J \mathrm{Ma}$ nipulative Physiol Ther. 2007;30(8):578-83.

35. Hou CR, Tsai LC, Cheng KF, Chung KC, Hong CZ. Immediate effects of various physical therapeutic modalities on cervical myofascial pain and trigger-point sensitivity. Arch Phys Med Rehabil. 2002;83(10):1406-14.

36. Hislop HJ, Montgomery J, Connolly BH.Daniels and Worthingham's muscle testing: techniques of manual examination.Philadelphia: WB Saunders; 1995. 